\title{
Experiencia con crosslinking en pacientes mexicanos con queratocono y variables queratométricas como factores pronóstico de agudeza visual
}

\author{
Experience with crosslinking in Mexican patients with keratoconus and keratometric \\ values as prognostic factors of visual acuity
}

\author{
Leopoldo Garduño-Vieyra1, Claudia R. González-Sánchez', Sergio E. Hernández-Da Mota ${ }^{2,3}$ y \\ $M^{a}$ Teresa Zavala-Martínez ${ }^{2,3 *}$ \\ 'Oftalmología Garduño, Clínica Oftalmológica, Guanajuato; ${ }^{2}$ Facultad de Medicina, Universidad Michoacana de San Nicolás de Hidalgo; ${ }^{3}$ Clínica \\ David, Unidad Oftalmológica. Morelia, Michoacán, México
}

\begin{abstract}
Resumen
Objetivo: El objetivo del estudio fue describir los resultados visuales y queratométricos de pacientes con queratocono sometidos a tratamiento con crosslinking (CXL). Método: Estudio retrospectivo de 277 ojos de 144 pacientes con queratocono progresivo que se sometieron a CXL. Se obtuvieron mediciones topográficas antes del procedimiento y 12 meses después. La agudeza visual corregida (AVC), la queratometría más plana (K1), la queratometría más curva (K2), el promedio de la queratometría (Kprom), el cilindro, el eje de K1, el eje de K2 y el valor paquimétrico del punto de la córnea más delgado fueron las variables evaluadas. Resultados: A los 12 meses de seguimiento, los valores de AVC mejoraron. Hubo disminuciones estadísticamente significativas en K1 $(p=0,001), K 2(p=0.000)$, eje de $K 1(p=0.000)$, eje de K2 $(p=0.004)$ y Krom ( $p=0.025)$. El análisis de correlación mostró que la AVC, la K1, la K2, el eje de K2, el Kprom y el cilindro preoperatorios, así como la K1, la K2, el Kprom y el cilindro posoperatorios, afectan significativamente la agudeza visual a los 12 meses de seguimiento. Conclusiones: El CXL es un tratamiento eficaz para mejorar la AVC a 1 año de seguimiento en el grupo de pacientes estudiados. Los valores de K preoperatorios y posoperatorios, y la AVC preoperatoria, se correlacionan de forma significativa con la AVC a los 12 meses de la aplicación del CXL.
\end{abstract}

PALABRAS CLAVE: Córnea. Queratocono. Riboflavina. Rayos ultravioleta. Crosslinking. Agudeza visual.

\begin{abstract}
Objective: The present study aimed to report the outcomes of patients with progressive keratoconus who were treated via conventional crosslinking (CXL). Method: This retrospective study included 277 eyes of 144 patients with progressive keratoconus who underwent CXL. Topographical measurements were obtained preoperatively and twelve months postoperatively. The best corrected visual acuity (BCVA), flat keratometry (K) value (K1), steep $K$ value (K2), average $K$ value (avgK), $K 1$ axis, K2 axis, pachymetry value and cylindrical value were the studied measures. Results: At the 12-month follow-up, the mean BCVA values were improved. Statistically significant decreases in K1 $(p=0,001), K 2(p=0.000), K 1$ axis $(p=0.000)$, $K 2$ axis $(p=0.004)$ and avgK $(p=0.025)$ were observed. The correlation analysis revealed that the preoperative BCVA, K1,
\end{abstract}

\section{Correspondencia:}

*María Teresa Zavala Martínez

Av. México 1020, Fracc. Américas Britania Fecha de recepción: 17-04-2018

C.P. 58270, Morelia, Mich., México Fecha de aceptación: 19-05-2018

E-mail: teisy_298@ hotmail.com

DOI: $10.24875 / C I R U .18000372$

Cir Cir. 2018;86:556-561

Contents available at PubMed www.cirugiaycirujanos.com 
K2, K2 axis, avgK and cylinder as well as postoperative K1, K2, avgK and cylinder values significantly affected visual acuity at the 12-month follow-up. Conclusions: CXL was an effective treatment to improve best corrected visual acuity. The preoperative and postoperative $K$ values, preoperative BCVA had a statistically significant correlation with BCVA at 12 months after CXL treatment.

KEY WORDS: Cornea. Keratoconus. Riboflavin. Ultraviolet rays. Crosslinking. Visual acuity.

\section{Introducción}

El queratocono es una enfermedad bilateral, asimétrica y degenerativa de la córnea, con una etiología desconocida que se caracteriza por una protrusión progresiva de la superficie corneal anterior y un adelgazamiento apical del estroma, lo que lleva a una importante morbilidad visual'. El procedimiento conocido como crosslinking $(\mathrm{CXL})$ produce la unión covalente entre las fibras de colágeno individuales para aumentar la rigidez del estroma corneal anterior, y se considera una de las más eficaces modalidades de tratamiento para detener o retrasar la progresión del queratocono ${ }^{2-4}$. En este tratamiento hay una reacción fotoquímica entre la riboflavina (vitamina B2) y la luz ultravioleta A (UVA); la riboflavina actúa como un fotosensibilizador para inducir el entrecruzamiento de las fibras de colágeno y como un escudo para proteger las demás estructuras intraoculares de daños por la luz UVA ${ }^{5}$. El CXL, al alterar la forma y la estructura de la córnea, puede llegar a mejorar la agudeza visual ${ }^{6}$.

Por otro lado, se sabe que el CXL puede ayudar a reducir los valores queratométricos basales. No obstante, los cambios en la córnea en el periodo posoperatorio no se han dilucidado completamente y requieren más estudios ${ }^{2-4}$. A pesar de que estudios anteriores han evaluado los cambios biomecánicos y clínicos en este sentido, los efectos de estos cambios en la agudeza visual se mantienen en cierta medida inciertos ${ }^{7}$. Este estudio tuvo como objetivo evaluar los resultados a los 12 meses en los pacientes tratados con CXL con queratocono progresivo y determinar la correlación entre las variables estudiadas.

\section{Método}

Este estudio retrospectivo fue aprobado por el comité de ética local y siguió los lineamientos de la declaración de Helsinki.

El queratocono progresivo se definió como un aumento de al menos 1.00 dioptrías en la medición de la queratometría más curva $(\mathrm{K})$ y la pérdida de al menos dos líneas de capacidad visual en el curso de 1 año. Los pacientes con retraso en la cicatrización epitelial, con una paquimetría corneal menor de 400 micras, mujeres embarazadas o en periodo de lactancia, con antecedentes de cirugía de córnea o de quemadura química, fueron excluidos del estudio.

Todos los pacientes fueron sometidos a CXL, que consistió en el siguiente procedimiento: en primer lugar se administró tetracaína tópica, se eliminaron $8.0 \mathrm{~mm}$ del epitelio central con el empleo de una espátula y a continuación se administró riboflavina $(0.1 \%$ en solución de dextrano al 20\%) por vía tópica cada 3 minutos durante 15 minutos. La córnea se centró y se expuso a un poder de emisión de luz UVA de $3 \mathrm{~mW} / \mathrm{cm}^{2}$ y una longitud de onda de $370 \mathrm{~nm}$ durante 30 minutos (Lightmed USA Inc., San Clemente, California, EE.UU.). La administración de riboflavina isotónica se continuó cada minuto durante la exposición a UVA. Después del tratamiento, la superficie del ojo se lavó con $20 \mathrm{ml}$ de solución salina balanceada. Al final del procedimiento se administró una combinación de antibiótico y lubricante tópico, y se colocó una lente de contacto blanda. El uso de esta lente de contacto se suspendió después del cierre del defecto epitelial.

La agudeza visual se determinó empleando la notación de Snellen, y posteriormente se realizó la conversión a unidades logMAR para el análisis estadístico. Se registró la capacidad visual de todos los pacientes antes del procedimiento y a los 12 meses de haberse realizado.

Antes de la operación y también a los 12 meses después del procedimiento se obtuvieron las siguientes mediciones topográficas empleando un topógrafo corneal CT-1000 (Towa Medical Instruments, Co., Ltd., Nakano, Nakanoshi Nagano, Japón): queratometría más plana (K1), eje de K1, queratometría más curva (K2), eje de K2, queratometría promedio (Kprom), valor del cilindro (astigmatismo topográfico) y grosor corneal de su zona más delgada (paquimetría) empleando un paquímetro ultrasónico (Pachmate DGH 55, DGH Technology, Inc., Exton, PA, EE.UU.). 


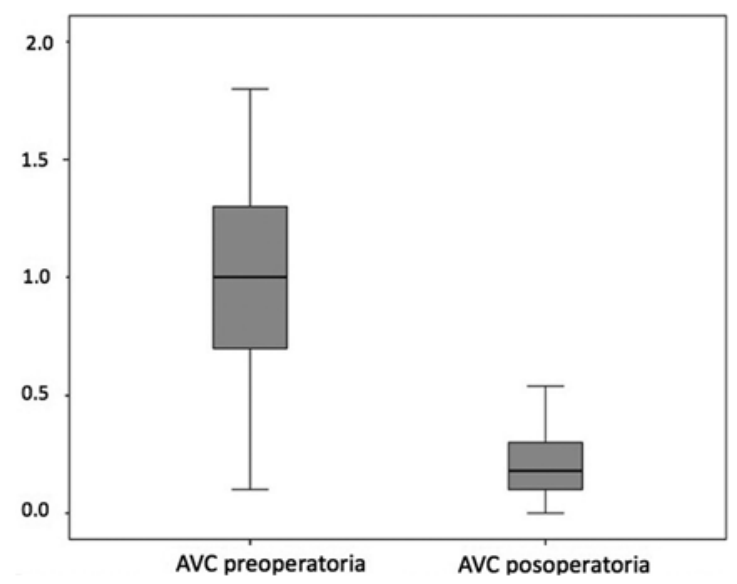

Figura 1. Gráfico de cajas que muestra la distribución de los valores de la agudeza visual corregida (AVC) preoperatoria y posoperatoria.

\section{Análisis estadístico}

Se utilizó el software SPSS (versión 21; IBM SPSS Statistics, Chicago, IL, EE.UU.) para el análisis estadístico de los resultados, los cuales se presentan como media \pm desviación estándar para las variables continuas. Se empleó la prueba t de Student pareada para la comparación de medias y se realizó un análisis de correlación para obtener el coeficiente de correlación de Pearson. Un valor de $p<0.05$ fue considerado como estadísticamente significativo.

\section{Resultados}

Se incluyeron 277 ojos de 144 pacientes (70 mujeres y 74 hombres; edad media: $27,03 \pm 8,65$ años) con queratocono progresivo que se sometieron a $\mathrm{CXL}$ entre julio de 2009 y enero de 2015 en Oftalmología Garduño, Clínica Oftalmológica de la Ciudad de León, Guanajuato, México.

La agudeza visual corregida (AVC) mostró una mejoría estadísticamente significativa al final del seguimiento, comparada con la agudeza visual preoperatoria $(p=0.000)$.

La paquimetría preoperatoria fue en promedio de 477.95 ( \pm 45.05$)$. No se tomó paquimetría posoperatoria. Los resultados de las variables queratométricas preoperatorias y al final del seguimiento se resumen en la tabla 1 y en las figuras 1 a 5 . La única variable que no mostró diferencias estadísticamente significativas entre el preoperatorio y el posoperatorio fue el valor del cilindro $(p=0.068)$.

La tabla 2 resume los resultados del análisis de correlación. La AVC, la K1, la K2, el eje de K2, el Kprom
Tabla 1. Diferencias entre el preoperatorio y el posoperatorio de las diferentes variables estudiadas

\begin{tabular}{lccc}
\hline & $\begin{array}{c}\text { Valores } \\
\text { preoperatorios }\end{array}$ & $\begin{array}{c}\text { Valores posoperatorios } \\
(\mathbf{1 2} \text { meses })\end{array}$ & p \\
\hline AVC (logMAR) & $1.12( \pm 0.44)$ & $0.18( \pm 0.12)$ & 0.000 \\
K1 (D) & $45.41( \pm 3.53)$ & $45.38( \pm 3.88)$ & 0.001 \\
K2 (D) & $50.36( \pm 5.60)$ & $50.02( \pm 4.51)$ & 0.000 \\
K1 eje & $168.65( \pm 50.02)$ & $153.96( \pm 58.83)$ & 0.000 \\
K2 eje & $99.01( \pm 44.47)$ & $104.09( \pm 52.33)$ & 0.004 \\
K & $47.82( \pm 5.13)$ & $47.60( \pm 5.23)$ & 0.025 \\
promedio (D) & & & \\
Cilindro (D) & $4.94( \pm 2.55)$ & $4.64( \pm 2.80)$ & 0.068 \\
\hline
\end{tabular}

AVC: agudeza visual corregida; logMAR: logaritmo del ángulo mínimo de resolución;

K1: queratometría más plana; K2: queratometría más curva; D: dioptrías

Tabla 2. Resultados del análisis de correlación entre las diferentes variables estudiadas y la agudeza visual corregida posoperatoria a los 12 meses

\begin{tabular}{lcc}
\hline AVC 12 meses & $\mathbf{r}$ & $\mathbf{p}$ \\
\hline Edad & 0.06 & 0.47 \\
AVC preoperatoria & 0.38 & 0.000 \\
K1 preoperatoria & 0.20 & 0.001 \\
K2 preoperatoria & 0.30 & 0.000 \\
Eje de K1 preoperatorio & -0.03 & 0.54 \\
Eje de K2 preoperatorio & -0.15 & 0.03 \\
K1 posoperatoria & 0.17 & 0.009 \\
K2 posoperatoria & 0.28 & 0.000 \\
Eje de K1 posoperatorio & -0.07 & 0.29 \\
Eje de K2 posoperatorio & -0.04 & 0.47 \\
Kprom preoperatorio & 0.27 & 0.000 \\
Kprom posoperatorio & 0.23 & 0.000 \\
Cilindro preoperatorio & 0.25 & 0.000 \\
Cilindro posoperatorio & 0.21 & 0.002 \\
Paquimetría & -0.11 & 0.66 \\
\hline AVC: agudeza visual corregida; K1: queratometría más plana: K2: queratometría más
\end{tabular}

AVC: agudeza visual corregida; K1: queratometría más plana; K2: queratometría más curva; Kprom: promedio de la queratometría

y el cilindro preoperatorios, así como la K1, la K2, el Kprom y el cilindro posoperatorios, afectan significativamente la agudeza visual a los 12 meses de seguimiento.

\section{Discusión}

En este estudio se analizaron los factores que afectan a la agudeza visual después de 12 meses de 


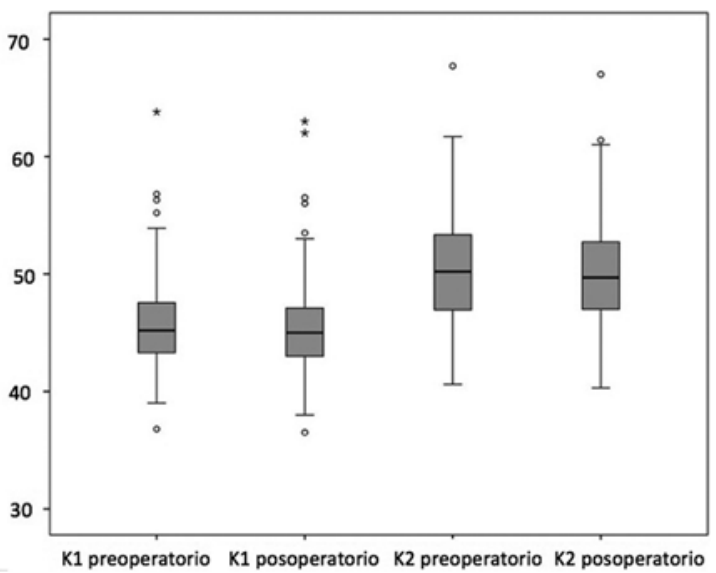

Figura 2. Gráfico de cajas que muestra la distribución de los valores de las queratometrías más planas (K1) y las queratometrías más curvas (K2) en dioptrías preoperatorias y posoperatorias.

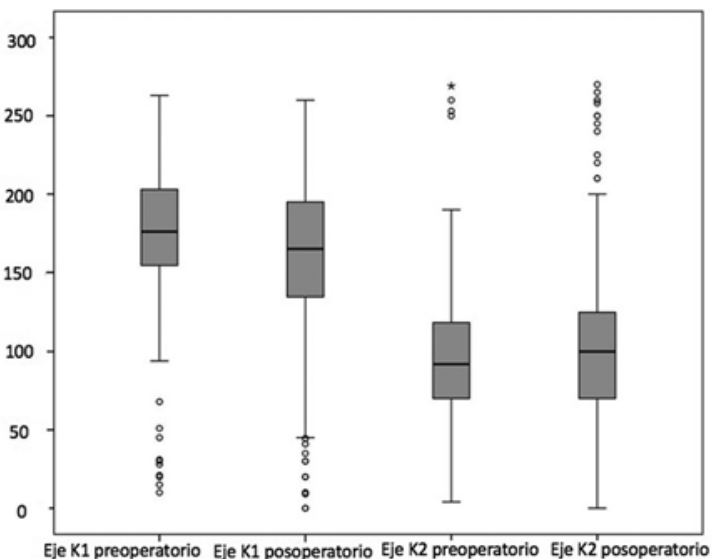

Figura 3. Gráfico de cajas que muestra la distribución de los valores de los ejes de las queratometrías más planas (K1) y de las queratometrías más curvas (K2) en dioptrías preoperatorias y posoperatorias.

seguimiento en pacientes tratados con CXL para queratocono progresivo, y se observó que la AVC, la K1, la K2, el eje de K2, el Kprom y el cilindro preoperatorios, así como la K1, la K2, el Kprom y el cilindro posoperatorios, tuvieron efectos significativos sobre la agudeza visual final. En particular, la agudeza visual mejoró significativamente después del tratamiento.

Aunque no se observaron diferencias estadísticamente significativas entre el preoperatorio y el posoperatorio en los valores del cilindro, sí las hubo en los demás valores, incluyendo los queratométricos, y muchas de las variables estudiadas afectaron la capacidad visual al final del seguimiento, excepto el eje de K1 preoperatorio, la K1 y la K2 posoperatorias, la edad y la paquimetría.

Destaca el hecho de que se observó una correlación negativa entre los valores del eje de K2 preoperatorio

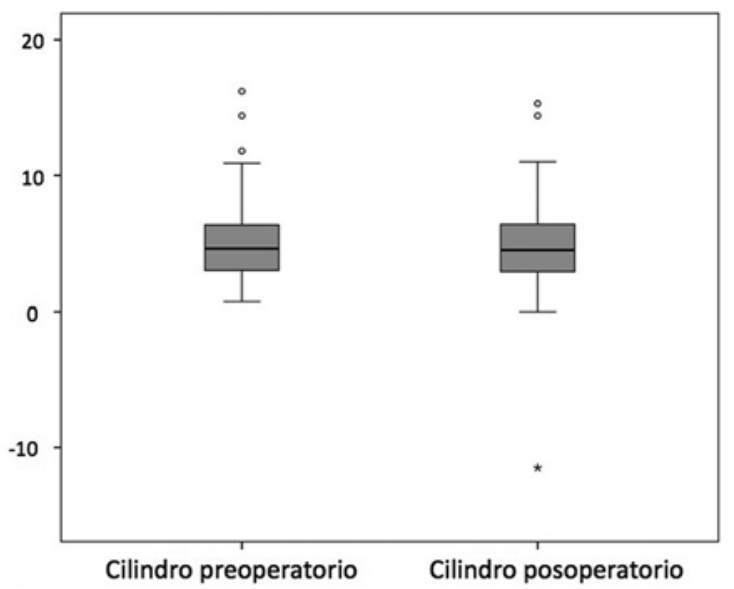

Figura 4. Gráfico de cajas que muestra la distribución de los valores cilíndricos preoperatorios y posoperatorios.

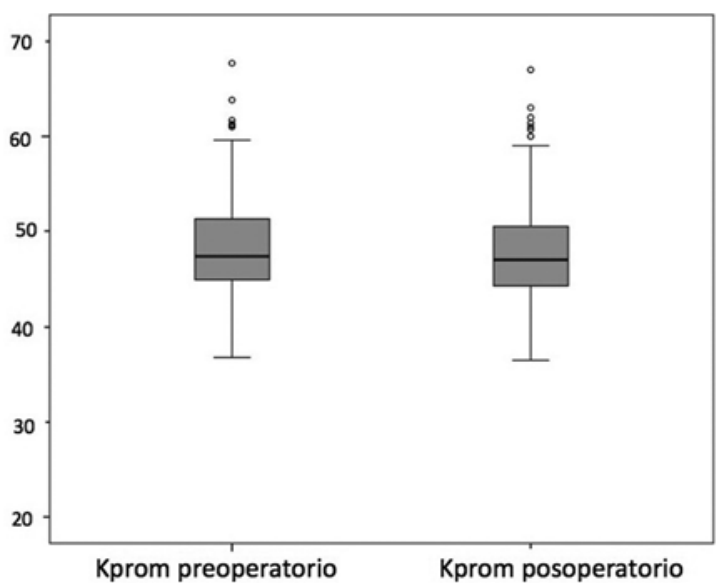

Figura 5. Gráfico de cajas que muestra la distribución de los valores de las queratometrías promedio (Kprom) en dioptrías preoperatorias y posoperatorias.

y la agudeza visual; en otras palabras, los menores valores del eje de $\mathrm{K} 2$ preoperatorios se asociaron con mejor agudeza visual a los 12 meses de seguimiento. Por otro lado, no se observó una correlación entre la paquimetría y la agudeza visual final.

Asimismo, las correlaciones fueron positivas entre la agudeza visual final y variables como la AVC preoperatoria, la K1 y la K2 preoperatoria, el eje de K2 preoperatorio, la K1 y la K2 posoperatoria, el Kprom y el cilindro preoperatorio y posoperatorio.

EI CXL del colágeno corneal aumenta la estabilidad biomecánica de la córnea y es el tratamiento preferido para el manejo del queratocono progresivo ${ }^{8}$. McAnena y $\mathrm{O}^{\prime} \mathrm{Keefe}^{9}$ describieron los resultados visuales, refractivos y topográficos siguientes al CXL en pacientes pediátricos con queratocono, y reportaron mejorías en la AVC y la $\mathrm{K}$ máxima, así como valores estables de la $\mathrm{K}$ mínima y el Kprom a 1 año. 
Sedaghat, et al. ${ }^{10}$ reportaron también mejorías estadísticamente significativas en la capacidad visual y la agudeza visual no corregida durante un seguimiento de 1 año después del CXL, junto con una disminución significativa en los valores queratométricos y del grosor del ápex corneal.

Hashemi, et al. ${ }^{11}$ compararon los resultados a los 6 meses del tratamiento con CXL acelerado del colágeno y con la modalidad estándar para el queratocono progresivo, y observaron que los cambios promedio en la agudeza visual sin corregir y la AVC, así como la disminución promedio en los valores de $\mathrm{K}$ máxima y de Kprom, no difirieron de manera estadísticamente significativa entre ambos grupos.

Berger, et al. ${ }^{12}$ reportaron la estabilización de la queratometría promedio y de la AVC en un niño tras 12 meses de seguimiento después de ser tratado con $\mathrm{CXL}$. Además de que estos resultados son similares a los nuestros, también respaldan el concepto de que el CXL posee efectos que limitan la progresión del queratocono.

Elbaz, et al. ${ }^{13}$ estudiaron retrospectivamente la eficacia del CXL acelerado en 16 ojos con queratocono, y reportaron la eficacia de este procedimiento en la estabilización de los parámetros topográficos, incluyendo la K1, la K2, el Kprom y el astigmatismo corneal, después de 12 meses de seguimiento. Mita, et al. ${ }^{14}$ evaluaron la eficacia del CXL acelerado en 39 ojos y observaron también una mejoría significativa de la agudeza visual no corregida y de la $\mathrm{K}$ máxima a los 6 meses del tratamiento.

Un hecho que cabe destacar es la correlación positiva que encontramos entre el eje de K2 preoperatorio y la AVC final, lo que no ha sido reportado, hasta donde sabemos, en otros estudios. Este hallazgo justifica una evaluación adicional y que se corrobore con estudios futuros.

Por otro lado, el valor máximo queratométrico se ha considerado como un indicador topográfico clave del éxito del CXL, y ha sido reportado como estable o disminuido después del CXL en algunos estudios ${ }^{14-16}$. Este parámetro no fue evaluado en nuestro estudio, siendo una de sus limitaciones.

En la actualidad, la información disponible sobre los factores que afectan el resultado del CXL es limitada. Destaca el hecho de que Toprak, et al. ${ }^{17}$ hayan reportado que, en los pacientes con queratocono progresivo, la edad, la agudeza visual y la paquimetría basales son factores que influyen en el éxito del tratamiento con CXL. En contraste, nosotros no encontramos que la edad afecte la agudeza visual final.
En su estudio empleando la técnica acelerada de CXL, Kirgiz, et al..$^{18}$ no encontraron diferencias estadísticamente significativas entre las mediciones preoperatorias y posoperatorias de los valores queratométricos, a diferencia de nosotros. Los resultados del análisis de correlación de su estudio son similares a los encontrados por nosotros.

De Angelis, et al..$^{19}$ reportaron una mejoría significativa en la AVC posoperatoria a 1 año, pero no un cambio significativo en la $\mathrm{K}$ máxima. También observaron que una menor agudeza visual preoperatoria, un astigmatismo alto refractivo y un queratocono avanzado son factores predictivos de una mejor AVC. Nuestros resultados concuerdan con los de ellos. En este sentido, observamos que hay una correlación positiva, por ejemplo, con el valor de K2 y el valor del cilindro, pero no con la AVC preoperatoria, que también, de acuerdo con nuestros hallazgos, tiende a correlacionarse positivamente con la AVC posoperatoria.

En lo que respecta a otras limitaciones de nuestro estudio, podemos señalar en primer lugar la falta de grupo control y de aleatorización, y el diseño prospectivo. También faltó tomar en cuenta algunas otras variables topográficas que pudieran correlacionarse con el resultado funcional del procedimiento en el corto y largo plazo, tales como los índices de simetría anterior y posterior, o el valor queratométrico máximo por topografía, entre otros. La K máxima, como se mencionó con anterioridad, es una variable importante para evaluar el éxito del tratamiento con CXL.

Por otro lado, los valores de queratometría se asociaron significativamente con el resultado visual, pero que se requiere el estudio de otras características o variables intervinientes o confusoras que pudieran explicar la mejoría visual. Atribuir el cambio de agudeza visual a los cambios queratométricos es algo que no se puede sustentar del todo con los resultados obtenidos en nuestro estudio. Son necesarios estudios más grandes y aleatorizados, con grupos control, tomando en cuenta variables confusoras y con periodos de seguimiento mayores, para definir mejor los factores que afectan los resultados del CXL.

En conclusión, el CXL fue un tratamiento eficaz para mejorar la AVC al inducir un aplanamiento corneal en esta serie de casos consecutivos de pacientes mexicanos, con mejoría en la capacidad visual.

\section{Conflicto de intereses}

Los autores declaran que no existe ningún conflicto de intereses. 


\section{Financiamiento}

El presente artículo no tuvo ninguna fuente de financiamiento.

\section{Bibliografía}

1. Jeyabalan N, Shetty R, Ghosh A, Anandula VR, Ghosh AS, Kumaramanickavel G. Genetic and genomic perspective to understand the molecular pathogenesis of keratoconus. Indian J Ophthalmol. 2013;61:384-8.

2. Ghanem RC, Santhiago MR, Berti T. Topographic, corneal wavefront, and refractive outcomes 2 years after collagen cross-linking for progressive keratoconus. Cornea. 2014;33:43-8.

3. Wollensak G. Crosslinking treatment of progressive keratoconus: new hope. Curr Opin Opthalmol. 2006;17:356-60.

4. Wollensak G, Spoerl E, Seiler T. Riboflavin/ultraviolet-A-induced collagen cross-linking for the treatment of keratoconus. Am J Ophthalmol. 2003;135:620-7.

5. Iseli HP, Thiel MA, Hafezi F, Kampmeier J, Seiler T. Ultraviolet A/riboflavin corneal cross-linking for infectious keratitis associated with corneal melts. Cornea. 2008;27:590-4.

6. Greenstein SA, Fry KL, Hersh PS. Corneal topography indices after corneal collagen crosslinking for keratoconus and corneal ectasia: oneyear results. J Cataract Refract Surg. 2011;37:1282-90.

7. Goldich Y, Marcovich AL, Barkana Y, Mandel Y, Hirsh A, Morad Y, et al Clinical and corneal biomechanical changes after collagen cross-linking with riboflavin and UV irradiation in patients with progressive keratoconus: results after 2 years of follow-up. Cornea. 2012;31:609-14.

8. Kanellopoulos AJ. Collagen cross-linking in early keratoconus with riboflavin in a femtosecond laser-created pocket: initial clinical results. J Refract Surg. 2009;25:1034-7.
9. McAnena L, O'Keefe M. Corneal collagen crosslinking in children with keratoconus. J AAPOS. 2015;19:228-32.

10. Sedaghat M, Bagheri M, Ghavami S, Bamdad S. Changes in corneal topography and biomechanical properties after collagen cross linking for keratoconus: 1-year results. Middle East Afr J Ophthalmol. 2015; 22:212-9.

11. Hashemi $H$, Fotouhi A, Miraftab M, Bahrmandy $H$, Seyedian MA, Amanzadeh K, et al. Short-term comparison of accelerated and standard methods of corneal collagen crosslinking. J Cataract Refract Surg. 2015;41:533-40.

12. Berger $Y$, Ezra-Nimni O, Skaat A, Fogel M, Grinbaum A, Barequet I. Corneal collagen cross-linking novel technique for prevention of keratoconus progression: results after one-year at the Sheba Medical Center. Harefuah. 2015;154:118-21.

13. Elbaz U, Shen C, Lichtinger A, Zauberman NA, Goldich Y, Chan CC, et al. Accelerated $\left(9-\mathrm{mW} / \mathrm{cm}^{2}\right)$ corneal collagen crosslinking for keratoconus-A 1-year follow-up. Cornea. 2014;33:769-73.

14. Mita M, Waring GO, Tomita M. High-irradiance accelerated collagen crosslinking for the treatment of keratoconus: six-month results. J Cataract Refract Surg. 2014; 40:1032-40.

15. Raiskup F, Theuring A, Pillunat LE, Spoerl E. Corneal collagen crosslinking with riboflavin and ultraviolet-A light in progressive keratoconus: ten-year results. J Cataract Refract Surg. 2015;41:41-6.

16. Derakhshan A, Shandiz JH, Ahadi M, Daneshvar R, Esmaily H. Shortterm outcomes of collagen crosslinking for early keratoconus. J Ophthalmic Vis Res. 2011;6:155-9.

17. Toprak I, Yaylalı V, Yildirim C. Factors affecting outcomes of corneal collagen crosslinking treatment. Eye (Lond). 2014;28:41-6.

18. Kirgiz A, Atalay K, Çabuk KŞ, Kaldirim H, Taşkapili M. Factors affecting visual acuity after accelerated crosslinking in patients with progressive keratoconus. Arq Bras Oftalmol. 2016;79:151-4

19. De Angelis F, Rateau J, Destrieux C, Patat F, Pisella PJ. Predictive factors for visual outcome after corneal collagen crosslinking treatment in progressive keratoconus: one-year refractive and topographic results. J Fr Ophtalmol. 2015;38:595-606. 